Mookherjea and colleagues ${ }^{8}$ have carefully examined the interplay of disorder and localization by fabricating a chain of 100 cuboidal resonators, connected by a single-mode photonic-wire waveguide on a silicon-on-insulator (SOI) wafer. The use of these small-format resonators enables the design of a compact device circumventing the need for optical gain media operating near $1,550 \mathrm{~nm}$, which are not readily available for SOI technology. In contrast to conventional slow-light structures, where tight fabrication tolerances are highly desirable, looser tolerances still allow a high degree of localization to be achieved in long waveguides. Based on theoretical considerations, the use of 100 unit cells is sufficient to observe Anderson localization of light with as little as $3 \%$ disorder, which can be achieved by inhomogeneity in the resonator parameters (Fig. 1b). This has not been observed previously because of the small number of resonators involved. If the resonators were identical (Fig. 1a), we would expect to see 'frozen light' with a standing wave pattern that extends across the entire structure. Though counterintuitive at first, with the introduction of disorder, which is in fact unavoidable in any fabrication process (Fig. 1b), we would expect to see propagating waves in which the intensity profile is localized over a few resonators in the waveguide.

Using a tunable continuous-wave laser to couple light into the photonic-wire waveguide, the team used a modified knife-edge detection scheme to measure the spatial distribution of light within the slow-light structure. An important feature of the fabricated structure, which had a band edge at about $1,575 \mathrm{~nm}$, was that no other slow-light modes existed within the 2-nm measurement window near the band edge. The smallest localization length, $L_{1}$, the authors measured was 6 unit cells (Fig. 1b), in excellent agreement with $L_{1}=5.6$ unit cells obtained from simulations ${ }^{8}$. More generally, $L_{1}$ was found to be inversely proportional to the square of the frequency, as expected with Anderson-type localization.

Given the widespread interest in exploring and exploiting devices in which the dispersion can be engineered to dramatically reduce the speed of light, the importance of the work presented by Mookherjea and colleagues ${ }^{8}$ lies in the demonstration that such structures are vulnerable to localization. This finding complements another recent description ${ }^{9}$ on the upper limit to the number of bit periods of time delay that can be achieved in a one-dimensional slow-light structure. Owing to these limitations, the challenge for researchers is to develop optimized devices and functionalities using both conventional materials and newly emerging metamaterials. In this context, future work with slow-light resonators that contain nonlinear optical gain media would be extremely interesting, as light localization may be altered by the nonlinear optical gain $^{10}$. At the present time it is not possible to predict the group velocity of light in such structures, and thus this research avenue remains wide open for future experimental and theoretical studies.

\footnotetext{
References

1. Hau, L. V., Harris, S. E., Dutton, Z. \& Behroozi, C. H. Nature 397, 594-598 (1999).

2. Liu, C., Dutton, Z., Behroozi, C. H. \& Hau, L. V. Nature 409, 490-493 (2001).

3. Bigelow, M. S., Lepeshkin, N. N. \& Boyd, R. W. Science 301, 200-202 (2003).

4. Vlasov, Y. A., O'Boyle, M., Hamann, H. F. \& McNab, S. J. Nature 438, 65-69 (2005).

5. Anderson, P. W. Philos. Mag. B 52, 505-509 (1985)

6. John, S. Phys. Rev. Lett. 53, 2169-2172 (1984)

7. Cao, H., Xu, J. Y., Seelig. E. W. \& Chang R. P. H. Appl. Phys. Lett. 76, 2997-2999 (2000).

8. Mookherjea, S., Park, J. S., Yang, S.-H. \& Bandaru, P. R. Nature Photon. 2, 90-93 (2008).

9. Miller, D. A. B. Phys. Rev. Lett. 99, 203903 (2007).

10. Schwartz, T., Bartal, G., Fishman, S. \& Segev, M. et al. Nature 446, 52-55 (2007).
}

\title{
CORRIGENDUM
}

\section{Cavities lead the way}

\section{MARKO LONCAR}

Nature Photonics 1, 565-567 (2007)

In this News \& Views article, on page 566, column 3, paragraph 1, line 10, the unit for input power was incorrect. The full sentence should have read:

For example, approximately $100 \mathrm{~kW}$ of optical power circulates in the cavity when only $1 \mathrm{~W}$ input power is injected into the fibre. 